\title{
Online assessment of cognitive functioning across the adult lifespan using the eCOGTEL: a reliable alternative to laboratory testing
}

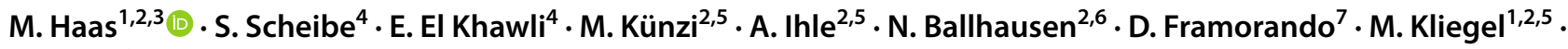 \\ S. Zuber ${ }^{2,5}$
}

Accepted: 19 November 2021 / Published online: 9 December 2021

(c) The Author(s), under exclusive licence to Springer Nature B.V. 2021

\begin{abstract}
As the population ages, risks for cognitive decline threaten independence and quality of life of older adults. Classically, psychological assessment tools that evaluate cognitive functioning are administered in face-to-face laboratory sessions, which are time- and resource-consuming. The present study set out to examine whether the eCOGTEL-an online adaptation of the Cognitive Telephone Screening Instrument (COGTEL; Kliegel et al. in J Psychol 141(2):147-170, 2007)—represents a reliable measure of cognitive performance in adulthood. Therefore, an age-stratified adult lifespan sample of 253 participants (aged 19-86 years) completed a face-to-face assessment in the laboratory and a self-administered online version, at their homes. A second, independent sample of 176 younger adults (aged 19-30 years) performed a test-retest assessment of the eCOGTEL. Results showed strong correlations between overall cognitive scores assessed online and in the laboratory, as well as a high test-retest reliability. Further, comparable data distributions between both assessment modes underline the feasibility of the eCOGTEL across the adult lifespan and particularly in older age. Our findings thereby indicate that the eCOGTEL can reliably measure cognitive performance across the lifespan at reduced costs, which may help detecting individuals at risk of developing age-related cognitive decline. Due to these strengths, the eCOGTEL represents a valuable contemporary approach for the resource-efficient online assessment of cognition, which may benefit a broad array of fundamental and applied research fields, such as clinical and organizational psychology.
\end{abstract}

Keywords Cognitive functioning $\cdot$ Screening instrument $\cdot$ Online assessment $\cdot$ Reliability $\cdot$ Lifespan

Responsible Editor: Marja J. Aartsen

\section{Haas}

Maximilian.Haas@unige.ch

1 Department of Psychology, Faculté de Psychologie et des Sciences de l'Education, University of Geneva, Boulevard du Pont d'Arve 28, CH-1205 Geneva, Switzerland

2 Centre for the Interdisciplinary Study of Gerontology and Vulnerability, University of Geneva, Geneva, Switzerland

3 Faculty of Psychology, Swiss Distance University Institute, Brig, Switzerland

4 University of Groningen, Groningen, The Netherlands

5 Swiss National Centre of Competences in Research LIVES - Overcoming vulnerability: life course perspectives, Lausanne, Geneva, Switzerland

6 Department of Developmental Psychology, Tilburg University, Tilburg, The Netherlands

7 School of Psychology, The University of Queensland, Brisbane, Australia

\section{Introduction}

Assessing cognitive functioning and its development across the adult lifespan plays an important role in multiple fields of human research and is highly relevant for clinical practice in particular. In fact, age-related cognitive decline is one of the most central aspects when studying aging societies, and maintaining one's level of cognitive functioning with advancing age will become even more pressing in a variety of applied contexts, such as the aging workforce (Beard and Bloom 2015; Fisher et al. 2017) or health promotion programs (Livingston et al. 2020; Wilkowska et al. 2018). Especially in the area of cognitive aging research, a particular focus is set on the development of cognitive abilities and their trajectories of functional change in healthy and clinical populations (Gerstorf et al. 2013; Sprague et al. 2020). Therefore, it is crucial to be able to assess, compare, and follow cognitive development with reliable and valid tools across the adult lifespan. With this study, we introduce and 
validate the eCOGTEL, an online version of the COGTEL (Kliegel et al. 2007), as a resource-efficient tool that allows researchers and practitioners to assess cognitive functioning in multiple domains.

\section{Laboratory assessments of cognitive functioning}

Up to today, a variety of assessment tools have been developed to assess cognitive functioning. Complex test batteries, such as the Wechsler Adult Intelligence Scale (WAISIV; Wechsler 2008), allow for a very detailed evaluation of participants' cognitive functioning, providing indicators not only of global cognitive performance, but also of the specific cognitive domains that are tested. However, extensive batteries administered in face-to-face assessments come along with multiple constraints, such as high demands in terms of resources (e.g., laboratory space, technical equipment, test material), personnel (e.g., trained staff for recruitment, testing, and evaluation), and time (e.g., recruitment, preparation, commuting to the laboratory). Such disadvantages make them less suitable for contexts with limited resources or for a large-scale application. In contrast to these intensive cognitive assessments, particularly in the area of life course epidemiology and cognitive aging research, screening instruments like the Mini-Mental State Examination (MMSE; Folstein et al. 1975) have been used extensively, as they have the advantage of providing a valid indicator of global cognitive functioning in a much shorter time while covering diverse domains (e.g., memory, language, orientation, and visual construction). Yet, the MMSE has been developed to screen for cognitive impairments (Mossello and Boncinelli 2006) and is therefore much less sensitive for differentiating individuals within the range of healthy cognitive functioning, both in older adults but even more so across the adult lifespan (Franco-Marina et al. 2010; Lopez et al. 2005). Although face-to-face laboratory assessments come with the benefits of a more controlled testing environment through direct contact with participants and possible oneto-one supervision, recent restrictions related to the global COVID-19 pandemic, for example, have illustrated that faceto-face data collection either can be complicated or even be made impossible due to factors that are out of researchers' control. Besides, studies that require participants to come into the laboratory systematically exclude participants with reduced mobility or scattered rural populations. Thus, there is a need for tools that can be used to assess cognitive functioning remotely.

\section{Assessing cognitive functioning outside of the laboratory}

Telephone-based evaluation constitutes a possible way to bypass some of the above-mentioned difficulties of classical face-to-face testing. In fact, research has proposed several of such instruments to assess different cognitive domains over the phone (for an overview, see Carlew et al. 2020; Castanho et al. 2014). It has been shown that most telephone-based assessments are consistent with inperson administration, particularly when used with typical populations (Hunter et al. 2021). However, it has also been suggested that a clinical application of such tools should reflect their specific diagnostic accuracy (Carlew et al. 2020; Elliott et al. 2020; Larner 2021). These mixed findings may be the result of the large variability in the overall purpose and target population of the assessments, but also of the cognitive functions that these instruments address, how they are designed (e.g., duration) and which outcome measures they offer (i.e., interpretation of global sum scores versus more specific scores for subscales). In this context, the Cognitive Telephone Screening Instrument (COGTEL) has been proposed as an assessment of global cognitive functioning via telephone (Kliegel et al. 2007). One strength of the COGTEL lies in the fact that it not only provides an overall score for general cognitive functioning, but also more detailed information on six cognitive domains that are central to cognition and relevant to everyday functioning while remaining relatively short to administer (approx. 15-20 min). Specifically, the COGTEL comprises subtests (and thus allows deriving separate scores) of verbal short-term and long-term memory, working memory, prospective memory, verbal fluency and inductive reasoning. In the initial study, Kliegel et al. (2007) validated the telephone-based tool with the face-toface administered version of the test and demonstrated that the COGTEL allows for measuring cognitive functioning validly via the telephone, outside of a standard laboratory test setting.

Subsequent research has confirmed the COGTEL as a valid and reliable assessment of cognitive functioning, also in terms of allowing a more fine-grained differentiation within the range of healthy cognitive development (Ihle et al. 2017). Moreover, several large-scale studies in epidemiological, aging, and pedagogical research have used the COGTEL so far (e.g., Breitling et al. 2010; Gouveia et al. 2020; Vallet et al. 2020), which further underlines its great flexibility and simple integration into a variety of research contexts.

Although telephone-based tools present certain advantages over laboratory assessments, other constraints remain unsolved, such as interferences due to hearing 
impairment with age, for example, or the (often difficultto-meet) requirement of a simultaneous presence of a trained assessment specialist and the participant. This can be particularly challenging when a large number of participants is tested, as it is the case in most epidemiological, individual differences and/or longitudinal studies. One way to address these constraints lies in developing a stand-alone version that does not require direct interaction with trained staff and that participants can complete at their own convenience, for example via the Internet. A self-administered online assessment may thus have the advantage of a better fit between motivational requirements and participants' objectives. Further, online testing may constitute a rapid and easy alternative to achieve a greater outreach to an even larger and more representative population, particularly in times in which research processes are slowed down due to external circumstances such as a pandemic.

In fact, past research using other tools has shown that web-based cognitive assessments can produce comparatively reliable results as their lab-based equivalents (e.g., Backx et al. 2020; Cromer et al. 2015). In their study, Backx and colleagues (2020) compared a web-based version to an inperson assessment of the Cambridge Neuropsychological Test Automated Battery (CANTAB; Sahakian et al. 1988). The CANTAB is a widely used tool in research and neuropsychological practice not only because of its sensitivity to detecting cognitive deficits and progressive decline in patients at early stages of Alzheimer's disease (Sahakian et al. 1988, 1990), but also due to its advantage of being a culture and language independent assessment. However, the latter may also be considered a disadvantage as it limits the CANTAB to a restricted set of cognitive functions (namely: visuospatial memory, attention, and emotional recognition), while excluding others that are central to global cognitive functioning (e.g., verbal fluency). Yet, considering a variety of cognitive domains is crucial to depicting global cognitive functioning in an appropriate manner. Further, since the in-person CANTAB has already been developed as a computerized test, its standardized modality of assessment does not only make it less sensitive to potential influences by an experimenter, but more generally renders the presence of the experimenter not essential. Therefore, contrary to the (e) COGTEL, one might expect differences between the webbased version and the in-person administration to be rather small by nature.

\section{Hypotheses and study goals}

The present study set out to examine whether an online version of the COGTEL would allow measuring global cognitive functioning across the adult lifespan in a valid and reliable way without the necessity of involving an experimenter. Specifically, the first research question addressed how well an online-administered version (eCOGTEL) maps with outcomes from the COGTEL administered in a standard face-to-face setting to evaluate global cognitive functioning in a sample covering the adult lifespan. More precisely, when administered in both settings, total scores should correlate to a high degree and means of both assessments should be comparable.

As a second aim, we particularly aimed at examining the validity of the eCOGTEL for older adults (defined as 60 years and older). Contrary to common assumptions that online assessments may be problematic for older populations, it has been suggested that they may provide a test environment that can have the advantage of reducing stress linked to elements of face-to-face testing (e.g., the presence of an experimenter, the fear of the imminent evaluation of one's cognitive abilities, or the feeling of being judged in case of low performance; Li et al. 2017; Sindi et al. 2013). Moreover, online assessments may help to solve some of the burdens linked to laboratory testing (e.g., difficulties in commuting to the lab) or telephone assessments (e.g., understanding instructions correctly via the phone). Yet, online testing may still represent a supplementary barrier to overcome for older adults. Even though computer usage becomes increasingly prominent among older adults, they still show great inequalities in their digital skills (Hargittai et al. 2019). Thus, frustration linked to physical or mental limitations among older adults with lower digital skills may have an impact on results (Gatto and Tak 2008); all rendering the issues of feasibility, relatability, and validity of a self-administered online cognitive assessment in older adults especially important.

\section{Method}

\section{Participants}

For the present study, an age-stratified adult lifespan sample of 262 French-speaking participants was recruited through advertisement in the wider area of Geneva. Nine participants were excluded either because their performance on cognitive background measures (described below) was $2.5 \mathrm{SD}$ below the norms $(n=6)$, or because they were considered an outlier with regard to their performance on the outcome measure (i.e., the absolute difference between laboratory and online score was greater than $2.5 \mathrm{SD}$ above the group mean $(n=3)$ ). Thus, the analyses presented in the following were conducted on a final sample of 253 participants $\left(M_{\text {age }}=46.80\right.$, $\mathrm{SD}=17.39,19-86$ years, $68 \%$ women). Table 1 shows sociodemographic characteristics of participants in the overall sample and for the subgroup of older adults. 
Table 1 Socio-demographic characteristics of participants

\begin{tabular}{|c|c|c|c|c|c|c|}
\hline & \multicolumn{3}{|l|}{ Overall sample } & \multicolumn{3}{|l|}{ Older adults only } \\
\hline & Total $(N=253)$ & $\begin{array}{l}\text { COGTEL first } \\
(n=141)\end{array}$ & $\begin{array}{l}\text { eCOGTEL first } \\
(n=112)\end{array}$ & Total $(N=72)$ & $\begin{array}{l}\text { COGTEL first } \\
(n=43)\end{array}$ & $\begin{array}{l}\text { eCOGTEL first } \\
(n=29)\end{array}$ \\
\hline $\mathrm{Age}^{\dagger}$ & $46.80(17.39)$ & $48.37(17.81)$ & $44.83(16.73)$ & $67.77(7.03)$ & $69.20(7.28)$ & $65.13(6.13)$ \\
\hline Age range & $19-86$ & $19-86$ & $19-82$ & $60-86$ & $60-86$ & $60-82$ \\
\hline Education (years) & $15.71(3.51)$ & $15.47(3.21)$ & $15.92(3.69)$ & $15.30(3.96)$ & $14.76(3.42)$ & $16.13(4.61)$ \\
\hline Mill Hill ${ }^{\dagger}$ & $24.50(4.65)$ & $24.08(4.76)$ & $25.04(4.46)$ & $26.39(4.37)$ & $25.51(4.61)$ & $27.75(3.66)$ \\
\hline Matrices & $18.93(4.45)$ & $18.90(4.29)$ & $18.97(4.68)$ & $16.04(5.09)$ & $15.95(4.81)$ & $16.18(5.57)$ \\
\hline Digit-symbol & $72.39(15.30)$ & $71.05(15.43)$ & $74.17(15.02)$ & $61.80(12.68)$ & $60.02(11.43)$ & $64.54(14.17)$ \\
\hline Computer literacy (CLS) ${ }^{\dagger}$ & $.77(0.11)$ & $.76(0.12)$ & $.77(0.09)$ & $.68(0.13)$ & $.66(0.14)$ & $.72(0.10)$ \\
\hline Typing speed (CPM) & $191.49(109.31)$ & $188.95(114.74)$ & $194.47(103.05)$ & $143.68(107.67)$ & $147.62(117.27)$ & $138.32(95.14)$ \\
\hline COGTEL total score s/i† $^{*}$ & $35.92(8.48)$ & $33.55(8.95)$ & $37.67(8.14)$ & $31.25(8.90)$ & $29.05(8.16)$ & $34.76(9.06)$ \\
\hline eCOGTEL total score & $34.87(10.16)$ & $34.65(10.11)$ & $35.23(10.27)$ & $28.71(9.90)$ & $27.91(10.60)$ & $29.84(8.90)$ \\
\hline
\end{tabular}

Results are presented as means with (Standard Deviations)

Mill Hill=raw scores; Matrices =age-norm standardized scores; Digit-symbol: age-norm standardized scores; $C L S$ computer literacy scale, $C P M$ characters typed per minute

Significant differences between administration orders are marked by an asterisk $(*)$ when referring to the overall sample, and by a dagger $(\dagger)$ when referring to the subsample of older adults

$* p<0.001$

${ }^{\dagger} p<0.05$

$\dagger p<0.01$

All participants gave informed consent to participate in the present study, which was approved by the Faculty Ethics Committee of the University of Geneva (decision protocol: PSE.20180803.02). Study enrollment was voluntary and participants were not remunerated; however, undergraduate psychology students $(n=38)$ were eligible to receive course credits for their participation.

\section{Materials and procedure}

\section{COGTEL and ECOGTEL}

Global cognitive functioning was assessed with the COGTEL, comprising six subtests that evaluate verbal short-term and long-term memory, working memory, prospective memory, verbal fluency and inductive reasoning. Specifically, for verbal short-term and long-term memory, participants were presented eight word-pairs that had to be recalled immediately after encoding, and after a delay of approximately 10-15 min, respectively. Four word-pairs were semantically associated (e.g., fruit-apple) and four were non-associated (e.g., salad-pen). The subtest on working memory consisted in the presentation of twelve series of digits that had to be recalled in reverse order right after presentation. The length of the series increased after every second series, from 2 to 7 digits that had to be retained and recalled at a time. For the subtest on verbal fluency, participants had to name as many words as possible that (a) began with a particular letter (i.e.,
A [or $S$, in the parallel version]; phonemic fluency), or (b) belonged to a particular category (i.e., professions [or furniture, in the parallel version]; semantic fluency). The number of produced words in both tasks was summed up to obtain the sub-score for verbal fluency. For the subtest on prospective memory, participants were instructed at the beginning of the COGTEL to name their year of birth at the beginning of the categorical fluency task. In the subtest on inductive reasoning, eight series of five numbers were presented, for which a sixth number had to be determined according to an underlying rule. For a more detailed description of the scales and administration, see Kliegel et al. (2007).

The COGTEL administered in a face-to-face laboratory setting was based on the validated version developed by Kliegel and colleagues (2007). The eCOGTEL, a contentwise equivalent online version of the instrument, was adapted as a tool running on any Internet browser via Qualtrics (Qualtrics, Provo, UT). All instructions and items were presented visually, thus avoiding reliance on additional audio equipment. Presentation times for all items as well as response times were kept identical to the faceto-face administration procedure. A total score was calculated for both the COGTEL and the eCOGTEL based on a weighted combination of the subtests that takes into consideration a comparable contribution of all six cognitive domains and simultaneously accounts for singlescale reliability (Kliegel et al. 2007): $7.2 \times$ prospective memory $+1.0 \times$ verbal short-term memory $+0.9 \times$ verbal 
long-term memory $+0.8 \times$ working memory $+0.2 \times$ verbal fluency $+1.7 \times$ inductive reasoning.

\section{Control measures}

Computer Literacy Scale As a potential confounding variable, participants' knowledge and expertise in using computer systems and interactive technology were assessed with the Computer Literacy Scale (CLS; Sengpiel and Dittberner 2008; Sengpiel and Jochems 2015). Part A of the CLS assesses general experience with computers ( 2 items: years of usage and hourly usage per week) as well as the diversity and intensity of activities (11 items; e.g., 'e-mail', 'text processing', 'online banking'). In Part B, which assesses objective computer knowledge, participants are presented with 26 items, representing either a symbol or a term that participants need to associate with its name or function (e.g., 'ESC button' meaning 'escape'; 'Browser' meaning 'a program to view websites on the Internet'). For both scales, participants' scores were divided by the maximum number of possible points, and computer literacy was calculated as the mean of the two scales on diversity and objective knowledge, ranging from 0 (no computer literacy) to 1 (high computer literacy).

Computer Typing Speed As an additional control measure, a typing speed task was administered at the beginning of the eCOGTEL procedure. Participants were instructed that they would see a short text which they should typewrite as fast as possible and without making any typing errors. The text consisted of 82 unrelated words of different lengths (500 characters in total) and the task stopped automatically after one minute, which resulted in the number of correctly typed characters per minute (CPM) as the control measure.

\section{Cognitive background measures}

Cognitive background measures included (a) the French version of the Mill Hill vocabulary scale as a measure of crystallized intelligence (Deltour 1993), (b) the matrices subtest as a measure of fluid intelligence, and (c) the digit-symbol subtest as a measure of processing speed (both taken from the Wechsler Adult Intelligence Scale-IV; Wechsler 2008).

\section{Procedure}

First, all participants came in for a short laboratory session covering socio-demographics, computer literacy, and cognitive background measures. At the end of the first session, participants received instructions for the further course of the study and fixed appointments for the following sessions. Within a delay of one to three days, participants either returned to the laboratory for the face-to-face administration of the COGTEL, or received an email with a link to the eCOGTEL that had to be completed within three days. Participants were instructed to do the eCOGTEL on a computer (as smartphones and tablets were not ideal for some response formats), in a quiet environment, and without possible disruptions through external influences such as telephone calls. Seven days later, they completed the other assessment mode of the test (eCOGTEL online or COGTEL in the laboratory, respectively). At the beginning of each version, participants were encouraged to complete the test without possible interruptions, but were informed that breaks could be arranged at any time if requested. For the eCOGTEL, the advancement between the different subtests was self-paced (i.e., the next stimuli were not presented unless the participant confirmed having understood the instructions and being ready to continue). The order of administration (i.e., face-to-face administration of the COGTEL and eCOGTEL) was counterbalanced, and all participants were randomly assigned to one of the two between-subject orders when enrolling in the study. The two groups did not differ on any of the demographic or cognitive background measures (all $p s>0.05$; see Table 1). Further, parallel versions of the test were used for the COGTEL and the eCOGTEL.

\section{Missing values}

Pairwise exclusion was applied for all analyses in the present study; therefore, participants with missing values on individual scales for either the COGTEL $(n=10)$ or the eCOGTEL $(n=22)$ were not excluded from the final sample. This concerned participants that did not complete their second session (COGTEL: $n=10$; eCOGTEL: $n=11$ ), that did not follow the instructed response format $(n=6)$, or that encountered technical problems with the eCOGTEL $(n=5)$ due to the Internet browser used.

\section{Sample and procedure for test-retest reliability of the eCOGTEL}

Test-retest reliability for the eCOGTEL was calculated with data coming from a second, independent sample of 176 young adults $\left(M_{\text {age }}=21.84, \mathrm{SD}=2.16,19-30\right.$ years, $81 \%$ women). All participants of this second sample were undergraduate psychology students that took part in an online study in exchange for course credits. Participants were asked to complete the eCOGTEL twice, with a delay of approximately one week between both sessions. More precisely, participants received an email with the link to the second session on the seventh day after their first assessment and were supposed to complete the second session within a delay of three days. Parallel versions of the test were used for the first and the second session. 


\section{Statistical analyses}

Concerning our main study aim, namely whether the eCOGTEL as a self-administered assessment evaluates global cognitive functioning reliably across the adult lifespan, Spearman correlation coefficients were calculated to evaluate the degree of agreement between participants' total scores and sub-scores for the two modes of assessments (laboratory vs. online; see Backx et al. 2020, for a similar procedure). We expected to observe correlations within a range similar to the ones reported by Kliegel and colleagues (2007) for the validation of the telephone-based version, i.e., around 0.70 or higher. Further, we calculated intraclass correlation coefficients (ICC) of total scores in the overall sample and the subsample of older adults. For both analyses, we expected that total scores should present an ICC $\geq 0.75$ to assure good reliability (Koo and Li 2016; Liljequist et al. 2019).

Mean differences between total scores of both assessments were expected not to be significantly different from zero, i.e., the null hypotheses were the desired outcome. Thus, complementary to the frequentist approach, we computed Bayes factors (BF) as an indicator of the degree to which the data support the null hypothesis rather than the alternative hypothesis (Kelter 2020; Masson 2011).

Further, regression analyses with age as a predictor were conducted separately for COGTEL and eCOGTEL total scores. Measures of computer literacy and participants' typing speed were added as potential confounders to account for differences in presentation modalities between both assessments.

\section{Results}

With regard to the first research question, results indicated that total scores of the two versions (COGTEL vs. eCOGTEL) were strongly positively correlated $\left(r_{\mathrm{s}}(223)=0.70\right.$, $p<0.001)$, whereas correlations between scores for the different subdomains were lower ( $r$ s ranging from $0.34-0.59$, all $p s<0.001$ ). Figure 1 shows univariate and bivariate distributions for total scores of both assessments, suggesting similar distributions between both assessment modes. Intraclass correlation showed moderate to good agreement for total scores of both assessments (ICC $(\mathrm{C}, 1)=0.69,95 \%$ CI $[0.62,0.76]$. Correlation coefficients for total scores as well as for the subdomains are shown in Table 2. A small but significant difference was found for COGTEL and eCOGTEL total scores $(\Delta M=1.05 ; t(222)=2.14, p=0.034$; for means, see Table 1). However, Bayesian analyses confirm anecdotal evidence in favor of the null hypothesis, i.e., no mean difference between total scores of both assessment modes $\left(\mathrm{BF}_{10}=0.70\right)$.

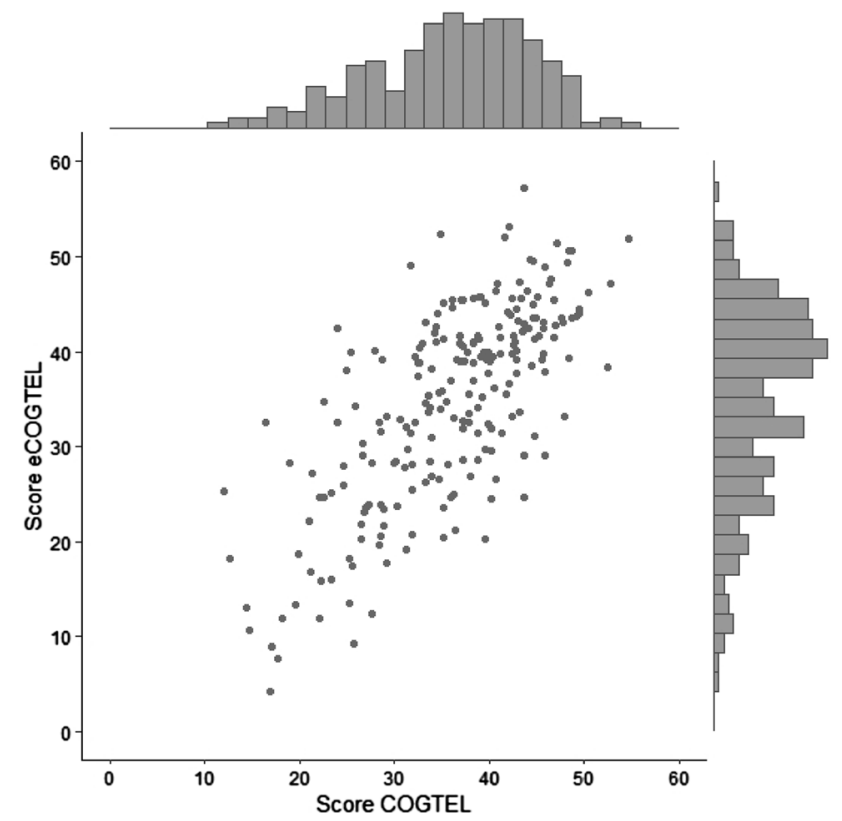

Fig. 1 Scatterplot and histograms of participants' total scores on the face-to-face COGTEL and the online-administered eCOGTEL.

Table 2 Correlations of COGTEL and eCOGTEL total scores and subdomain scores

\begin{tabular}{llll}
\hline & Overall & COGTEL first & eCOGTEL first \\
\hline Total score & 0.70 & 0.71 & 0.70 \\
Verbal short-term & 0.39 & 0.45 & 0.33 \\
$\quad$ memory & & & \\
Working memory & 0.39 & 0.31 & 0.48 \\
Verbal fluency & 0.34 & 0.33 & 0.35 \\
Prospective memory & 0.36 & 0.45 & 0.26 \\
Inductive reasoning & 0.51 & 0.45 & 0.59 \\
Verbal long-term memory & 0.50 & 0.51 & 0.49 \\
\hline
\end{tabular}

All correlations were significant at $p<0.001$

Moreover, we observed a significant difference in COGTEL total scores depending on whether participants did the COGTEL before or after the eCOGTEL $(t(241)=-3.69$, $\left.p<0.001 ; \mathrm{BF}_{10}=76.61\right)$, whereas no significant order effect was observed for eCOGTEL total scores $(p>0.05$; $\left.\mathrm{BF}_{10}=0.16\right)$.

Further, regression analyses with age as a predictor were conducted separately on COGTEL and eCOGTEL total scores as outcome measures. In a first linear regression analysis, age was entered as the only predictor and significantly predicted COGTEL total scores, $\beta=-0.41$, $t(241)=-7.02, p<0.001$. As expected, an increase in age predicted a lower total score in the face-to-face assessment, $R^{2}=0.17, F(1,241)=49.27, p<0.001$. Including the order of presentation as an additional predictor in a second step 
showed that a later presentation order predicted higher performance on the COGTEL, $\beta=-0.17, t(212)=-6.34$, $p=0.007, \Delta R^{2}=0.03$.

To account for the online-based presentation, a hierarchical regression with age as a single predictor in a first step, age and presentation order in a second step, and typing speed (CPM) and computer literacy (CLS) as additional predictors in a third step, was conducted on the eCOGTEL total score. These analyses confirmed age to be a significant negative predictor of the eCOGTEL total score over and above CPM and CLS $(\beta=-0.24, t(207)=-3.91, p<0.001)$. An increase in age significantly predicted a lower performance also on the eCOGTEL, $R^{2}=0.26, F(1,210)=73.82$, $p<0.001$, and the amount of variance explained by age was similar to what was found for the face-to-face assessment $(z=-1.314, p=0.09)$. Further, both CPM $(\beta=0.37$, $t(208)=6.45, p<0.001)$ and CLS $(\beta=0.25, t(208)=4.04$, $p<0.001)$ significantly predicted performance on the eCOGTEL, $\Delta R^{2}=0.20, F(2,207)=37.95, p<0.001$. Thus, a higher number of characters typed per minute as well as a higher computer literacy each predicted a better total score on the eCOGTEL. Presentation order was not a significant predictor of eCOGTEL total scores, $\beta=-0.07, t(209)=-1.24$, $p=0.21, \Delta R^{2}=0.01$. Figure 2 shows the distribution of COGTEL total scores (left) and eCOGTEL total scores (right) as well as their regression on age across the adult lifespan. To examine the impact of administration order, computer skills, and demographic data on test-retest variance in subtest performance, hierarchical regressions were conducted on subtest score differences between COGTEL and eCOGTEL (refer to Supplement Table A).
As described above, test-retest reliability of eCOGTEL scores was calculated with data from a second, independent sample of younger adults who completed the onlineadministered format twice with a delay of one week between assessments. eCOGTEL total scores were highly correlated, $r=0.71, p<0.001$, and presented a moderate to good test-retest reliability, $\mathrm{ICC}(\mathrm{C}, 1)=0.70,95 \% \mathrm{CI}[0.61,0.77]$. Further, total scores did not differ significantly between test and retest $(t(165)=-1.04, p=0.30)$, and Bayesian analysis confirmed moderate evidence in favor of the null hypothesis $\left(\mathrm{BF}_{10}=0.15\right)$. Table 3 shows reliability scores for the eCOGTEL total score as well as for each of the subdomains ( $r$ s ranging from 0.42 to 0.66 , all $p s<0.001$ ).

Table 3 Descriptive statistics and test-retest reliability of eCOGTEL total score and subdomain scores in the secondary sample of 176 young adults

\begin{tabular}{|c|c|c|c|c|c|}
\hline & \multicolumn{2}{|l|}{ Test } & \multicolumn{2}{|l|}{ Retest } & \multirow[t]{2}{*}{ Reliability $r$} \\
\hline & $M$ & $S D$ & $M$ & $S D$ & \\
\hline Total score & 40.27 & 7.07 & 40.54 & 6.71 & $0.70 * * *$ \\
\hline Verbal short-term memory & 6.40 & 1.56 & 5.90 & 1.88 & $0.42 * * *$ \\
\hline Working memory & 8.70 & 3.02 & 8.91 & 3.17 & $0.47 * * *$ \\
\hline Verbal fluency & 28.03 & 6.45 & 24.83 & 5.99 & $0.66^{* * *}$ \\
\hline Prospective memory & 0.77 & 0.42 & 0.91 & 0.28 & $0.42 * * *$ \\
\hline Inductive reasoning & 5.38 & 2.05 & 5.95 & 1.93 & $0.53 * * *$ \\
\hline Verbal long-term memory & 7.23 & 1.02 & 6.49 & 1.82 & $0.41 * * *$ \\
\hline
\end{tabular}

Except for Total Score and Working Memory, all other means were significantly different between test and retest $(p s<0.001)$

$* * *$ significant at $p<0.001$
Fig. 2 Distributions of COGTEL total scores (A) and eCOGTEL total scores (B) as well as their regression on age. Note. Both regression lines represent simple linear regressions with age as a single predictor. The amount of variance explained by age was comparable in both models
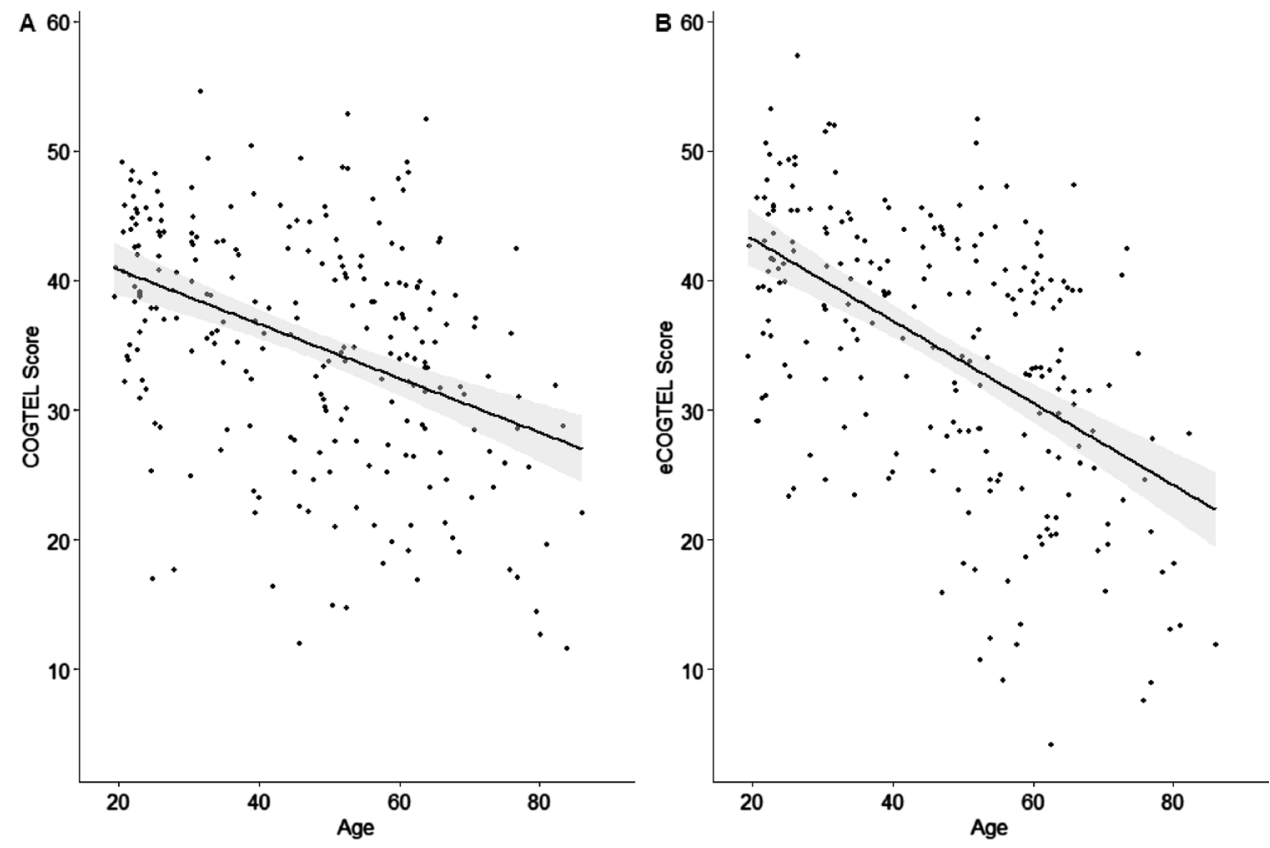

Note. Both regression lines represent simple linear regressions with age as a single predictor. 


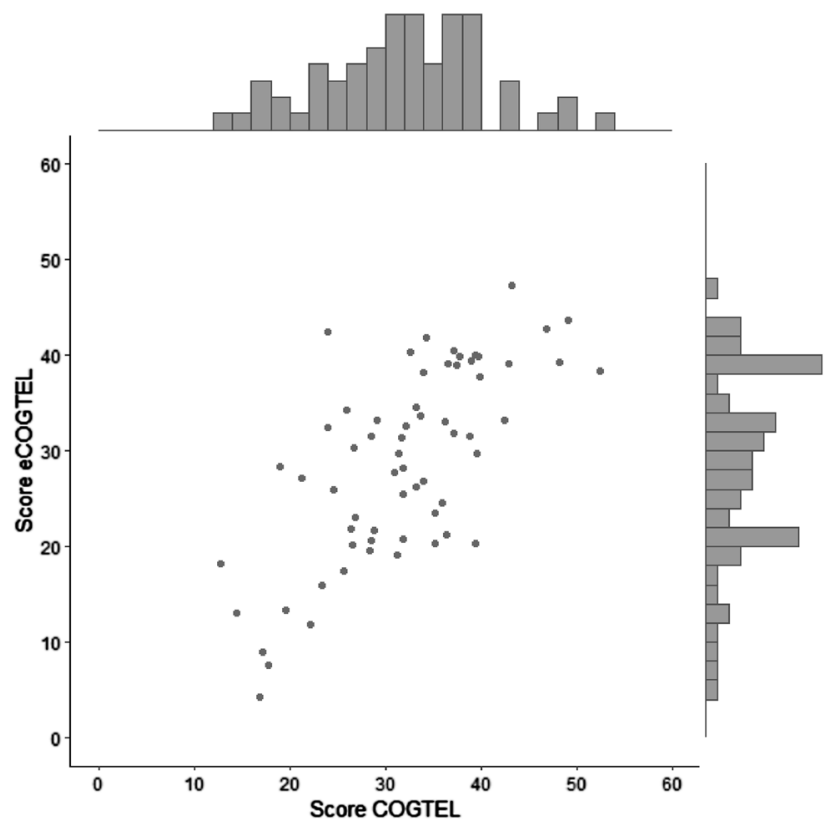

Fig. 3 Scatterplot and histograms of participants' total scores on the face-to-face COGTEL and the online-administered eCOGTEL for older adult subsample (60 years and older)

Table 4 Correlations of COGTEL and eCOGTEL total scores and subdomain scores for the older adult subsample (60 years and older)

\begin{tabular}{llll}
\hline & Overall & COGTEL first & eCOGTEL first \\
\hline Total score & $0.71^{* * *}$ & $0.70^{* * *}$ & $0.75^{* * *}$ \\
Verbal short-term & $0.36^{* * *}$ & $0.46^{* * *}$ & $0.19 \mathrm{~ns}$ \\
$\quad$ memory & & & \\
Working memory & $0.36^{* * *}$ & $0.30 \mathrm{~ns}$ & $0.48^{*}$ \\
Verbal fluency & $0.68^{* * *}$ & $0.57 * * *$ & $0.77^{* * *}$ \\
Prospective memory & $0.40^{* * *}$ & $0.36^{*}$ & $0.46^{*}$ \\
Inductive reasoning & $0.54^{* * *}$ & $0.46^{* * *}$ & $0.64 * * *$ \\
$\quad \begin{array}{l}\text { Verbal long-term } \\
\text { memory }\end{array}$ & $0.45^{* * *}$ & $0.49^{* * *}$ & $0.31 \mathrm{~ns}$ \\
\hline
\end{tabular}

$\mathrm{ns}=$ not significant

*significant at $p<0.05$.

**significant at $p<0.001$

With regard to the second research question, namely whether the eCOGTEL constitutes an adequate assessment of global cognitive functioning in older adults despite additional hurdles through technology-based presentation, the following in-depth analyses were conducted on the subsample of adults aged 60 years and above only $(n=72)$. Figure 3 shows the univariate and bivariate distributions of total scores on the face-to-face COGTEL and the selfadministered eCOGTEL in older adults. Correlations of older adults' total scores and of their scores in each subdomain are presented in Table 4. Similar to what was found for the full sample, a higher performance on the COGTEL was associated with a higher performance on the eCOGTEL also in the subgroup of older adults $\left(r_{\mathrm{s}}(64)=0.71, p<0.001\right)$. Intraclass correlation showed moderate to good agreement for total scores of both assessments, $\operatorname{ICC}(C, 1)=0.66,95 \%$ CI $[0.46,0.79]$.

Moreover, comparisons of mean total scores revealed a significant difference between both assessments, indicating that, overall, older adults presented higher total scores on the COGTEL than on the eCOGTEL $(\Delta M=2.54 ; t(63)=3.45$, $p=0.001$; for means, see Table 1). However, as was the case for the overall sample, we observed a significant order effect on COGTEL total scores $(t(68)=2.73, p=0.008$; $\left.\mathrm{BF}_{10}=5.54\right)$. Older adults who performed the eCOGTEL first subsequently had higher COGTEL scores compared to those who started with performing the COGTEL. In contrast, no significant order effect was observed for eCOGTEL total scores $\left(t(63)=0.77, p=0.44 ; \mathrm{BF}_{10}=0.33\right)$. Further, no significant difference was obtained between COGTEL and eCOGTEL total scores for participants that completed the face-to-face assessment first $(t(37)=1.43, p=0.16$; $\left.\mathrm{BF}_{10}=0.45\right)$.

Further, we conducted the same regression analyses as for the overall sample specifically for older adults. In a first linear regression analysis, age was entered as the only predictor and significantly predicted COGTEL total scores, $\beta=-0.49$, $t(68)=-4.58, p<0.001$. As expected, an increase in age predicted a lower total score in the face-to-face assessment, $R^{2}=0.24, F(1,68)=20.94, p<0.001$.

For the eCOGTEL total score, age did not remain a significant predictor after adding CPM and CLS to the analyses $(\beta=-0.16, t(51)=-1.46, p=0.15)$. However, both CLS $\left(\beta=0.37, t(51)=3.34, p=0.002, \Delta R^{2}=0.16\right)$ and CPM $\left(\beta=0.41, t(51)=3.72, p<0.001, \Delta R^{2}=0.15\right)$ significantly predicted performance on the eCOGTEL in older adults.

\section{Discussion}

The aim of the present study was to investigate the reliability of the eCOGTEL as an online-administered assessment of global cognitive functioning across the lifespan, which has been confirmed. Specifically, COGTEL and eCOGTEL total scores strongly correlated and this correlation was comparably high as the one between the face-to-face and the telephonebased version of the instrument (approx. $r=0.70$ in both studies) initially reported by Kliegel and colleagues (2007). Moreover, comparable distributions of total scores were observed in both assessment formats. Importantly, total scores did not present any floor or ceiling effects, neither in the laboratory, nor in the online setting. This corroborates evidence that the instrument allows discriminating well across the full range of cognitive performances in both assessment settings. Yet, 
a slightly higher variance in performance was observed for the eCOGTEL. One possible explanation for this could lie in differences in stimuli presentation and/or their processing. In fact, all stimuli were presented visually in the eCOGTEL and responses had to be typed, whereas stimuli and responses in the face-to-face setting were both given verbally. Despite these differences, total scores of both assessment modes were statistically almost identical.

Test-retest reliability of the eCOGTEL was high for the total score, but was somewhat lower for the different subdomain scores, which corresponds to the patterns reported by Kliegel et al. (2007) for the telephone-based version. Interestingly, compared to total scores in the face-to-face assessment, eCOGTEL total scores did not vary significantly between counterbalancing orders (i.e., whether the eCOGTEL was completed before or after the COGTEL). However, total scores on the COGTEL were higher for participants that completed the eCOGTEL first, suggesting a possible practice effect. In fact, the face-to-face assessment seems to be affected more strongly by retest effects, whereas the eCOGTEL total score seems to be less impacted and was more stable over time. With regard to the subtests, no clear trend could be observed for the impact of administration order, computer skills, or demographic data, and the variance explained by these factors was generally rather small. These findings further underline that an online-administered assessment of cognitive functioning with the eCOGTEL represents a reliable alternative to a face-to-face evaluation.

In a second research question, the present study investigated whether the eCOGTEL constitutes an adequate assessment of cognitive functioning particularly in older adults (aged 60 years and older) despite the possible technologyrelated challenges presented by an online format. Results showed that the correlation between older adults' total scores was identical to the one of the full sample with the full age range. Moreover, scores in both settings were welldistributed and older adults' performances did not present any floor or ceiling effects. This does not only confirm that the eCOGTEL assesses global cognitive functioning reliably also in older adults, but it shows more generally that with the increasing digitalization of older adults (Federal Statistical Office 2020; Hunsaker and Hargittai 2018), online tools may constitute a possible way to assess cognitive performance validly in an aging population (Myers et al. 2016).

\section{Limitations and outlook}

Although test-retest reliability of the overall score was high, it was generally low (and below acceptable levels) for the different subdomain scores of the eCOGTEL, which makes it difficult to make solid conclusions on the cognitive domains tested. One possible explanation could lie in the short delay of administration between both sessions (approximately one week). Participants were likely to remember the structure of the assessment and its different components, which may have favored the use of strategies (e.g., focusing on subtests that were performed less well or perceived as more difficult in the first session, while putting less effort into subtests that seemed easier to solve). Interestingly, the pattern is rather inconsistent between subdomains that gain in performance (Prospective Memory, Inductive Reasoning) and those that show losses across time (Verbal Short-/Long-Term Memory, Verbal Fluency). Only the subscale on working memory did not show any significant differences between test and retest. Future research would need to further investigate the psychometric properties of the eCOGTEL subdomains as well as the factors that may have an impact on performance. Further, it would be interesting to investigate test-retest reliability with longer delays between assessments. As total scores for both COGTEL and eCOGTEL were computed with the same formula, future studies could investigate whether one may consider revising and adapting the formula to acknowledge for these differences in the eCOGTEL assessment. This may be of particular importance for subtests that request production of more than a single response, such as in the verbal fluency task. In the present study, we accounted for differences in presentation by controlling for participants' computer skills. However, Stringer and colleagues (2018) have shown in a group of healthy and cognitively impaired older adults that features such as slower typing are associated with cognitive functioning, particularly with memory. Indeed, in the present study, older adults had lower performance on the (e) COGTEL and showed lower computer literacy than younger adults. Thus, online testing may still represent a challenge for those older adults who are less familiar with computer technology. Yet, recent demographic studies have shown that more and more older adults have access to and are at ease with using computer technology. Future studies will have to examine in more detail how well online tools work for older adults, particularly for the oldest (i.e., 75 years and more). Thus, future studies may systematically address measures of computer skills when possibly revising the formula of the eCOGTEL rather than merely considering them as control variables. This could be achieved by adding a fixed coefficient to the computed total score, or by specifically performing adjustments on the subdomains that seem to rely on computer skills more than others (see Supplement Table A).

As mentioned above, the COGTEL has been used in various international research projects and different language versions exist already (German, English, French, and Portuguese). By making all material and the eCOGTEL demo version accessible online, we encourage future research to use and adapt the tool to their specific needs. Moreover, with regard to the advancing digitalization of society, it may be worth investigating a version that can be run on 
portable devices such as smartphones or tablets. Although we did not find any evidence suggesting that there may have been a difference in acceptance (i.e., comparable number of incomplete data and dropouts in both settings), future studies should more systematically address the acceptance of formats adapted from standard laboratory assessments.

\section{Conclusion}

Taken together, the present study demonstrates that assessing global cognitive functioning online with the eCOGTEL is feasible across the adult lifespan and presents a reliable and valid measure of cognitive performance. Moreover, we specifically confirm this for the older adults' age range. Thus, due to these strengths, online assessment of cognition is not only perfectly in line with the zeitgeist of the study of cognitive aging, but a valuable contemporary approach for a broad array of applied research fields such as clinical and organizational psychology.

Supplementary Information The online version contains supplementary material available at https://doi.org/10.1007/s10433-021-00667-x.

Acknowledgements We thank Estelle Gillioz, Vanessa Garcia, Annick Hottelier, Laurène Kuhn, Clémentine Laboret, Laura Pede, Hadir Elhanafi, Clara Moulin, and Malory Sugnaux for their assistance in study preparation and data collection. This work was supported by the Swiss National Science Foundation (SNSF) under Grant 100019_165572 and by the Swiss National Center of Competences in Research LIVES-Overcoming vulnerability: life course perspectives, which is financed by the SNSF (Grant Number: 51NF40-160590).

Data availability Material and instructions for the COGTEL and a demo version of the eCOGTEL will be made available on the institutional website (https://cigev.unige.ch/openscience) in all available languages.

\section{Declarations}

Conflict of interest The authors have no conflict of interest to declare.

\section{References}

Backx R, Skirrow C, Dente P, Barnett JH, Cormack FK (2020) Comparing web-based and lab-based cognitive assessment using the Cambridge Neuropsychological Test Automated Battery: a withinsubjects counterbalanced study. J Med Internet Res 22(8):e16792. https://doi.org/10.2196/16792

Beard JR, Bloom DE (2015) Towards a comprehensive public health response to population ageing. Lancet 385(9968):658-661. https:// doi.org/10.1016/S0140-6736(14)61461-6

Breitling LP, Wolf M, Müller H, Raum E, Kliegel M, Brenner H (2010) Large-scale application of a telephone-based test of cognitive functioning in older adults. Dement Geriatr Cognit Disord 30(4):309-316. https://doi.org/10.1159/000319896
Carlew AR, Fatima H, Livingstone JR, Reese C, Lacritz L, Pendergrass C, Bailey KC, Presley C, Mokhtari B, Cullum CM (2020) Cognitive assessment via telephone: a scoping review of instruments. Arch Clin Neuropsychol 35(8):1215-1233. https://doi.org/10. 1093/arclin/acaa096

Castanho TC, Amorim L, Zihl J, Palha JA, Sousa N, Santos NC (2014) Telephone-based screening tools for mild cognitive impairment and dementia in aging studies: a review of validated instruments. Front Aging Neurosci. https://doi.org/10.3389/fnagi.2014.00016

Cromer JA, Harel BT, Yu K, Valadka JS, Brunwin JW, Crawford CD, Mayes LC, Maruff P (2015) Comparison of cognitive performance on the cogstate brief battery when taken in-clinic, in-group, and unsupervised. Clin Neuropsychol 29(4):542-558. https://doi.org/ 10.1080/13854046.2015.1054437

Deltour JJ (1993) Echelle de vocabulaire de Mill Hill de JC Raven. Adaptation française et normes européennes du Mill Hill et du Standard Progressive Matrices de Raven (PM38)

Elliott E, Green C, Llewellyn DJ, Quinn TJ (2020) Accuracy of telephone-based cognitive screening tests: systematic review and meta-analysis. Curr Alzheimer Res 17(5):460-471. https://doi. org/10.2174/1567205017999200626201121

Federal Statistical Office (2020) Digitale Kompetenzen. https://www. bfs.admin.ch/bfs/de/home/statistiken/kultur-medien-informatio nsgesellschaft-sport/informationsgesellschaft/gesamtindikator en/haushalte-bevoelkerung/digitalekompetenzen.html. Accessed 12 Jan 2021

Fisher GG, Chaffee DS, Tetrick LE, Davalos DB, Potter GG (2017) Cognitive functioning, aging, and work: a review and recommendations for research and practice. J Occup Health Psychol 22(3):314-336. https://doi.org/10.1037/ocp0000086

Folstein MF, Folstein SE, McHugh PR (1975) "Mini-mental state": a practical method for grading the cognitive state of patients for the clinician. J Psychiatr Res 12(3):189-198

Franco-Marina F, García-González JJ, Wagner-Echeagaray F, Gallo J, Ugalde O, Sánchez-García S, Espinel-Bermúdez C, Juárez-Cedillo T, Rodríguez MÁV, García-Peña C (2010) The Mini-Mental State Examination revisited: ceiling and floor effects after score adjustment for educational level in an aging Mexican population. Int Psychogeriatr 22(1):72-81. https://doi.org/10.1017/S104161020 9990822

Gatto SL, Tak SH (2008) Computer, internet, and E-mail use among older adults: benefits and barriers. Educ Gerontol 34(9):800-811. https://doi.org/10.1080/03601270802243697

Gerstorf D, Ram N, Lindenberger U, Smith J (2013) Age and time-todeath trajectories of change in indicators of cognitive, sensory, physical, health, social, and self-related functions. Dev Psychol 49(10):1805-1821. https://doi.org/10.1037/a0031340

Gouveia BR, Gouveia ÉR, Kliegel M, Lopes H, Rodrigues A, Marques A, Correia AL, Alves R, Ihle A (2020) Face-to-face assessment of COGTEL in adolescents: test-retest reliability and association with school grades. Revista Latinoam Psicolo 52:169-175. https:// doi.org/10.14349/rlp.2020.v52.17

Hargittai E, Piper AM, Morris MR (2019) From internet access to internet skills: digital inequality among older adults. Univers Access Inform Soc 18(4):881-890. https://doi.org/10.1007/ s10209-018-0617-5

Hunsaker A, Hargittai E (2018) A review of Internet use among older adults. New Media Soc 20(10):3937-3954. https://doi.org/10. $1177 / 1461444818787348$

Hunter MB, Jenkins N, Dolan C, Pullen H, Ritchie C, Muniz-Terrera G (2021) Reliability of telephone and videoconference methods of cognitive assessment in older adults with and without dementia. J Alzheim Dis 81(4):1625-1647. https://doi.org/10.3233/ JAD-210088

Ihle A, Gouveia ÉR, Gouveia BR, Kliegel M (2017) The cognitive telephone screening instrument (COGTEL): a brief, reliable, and 
valid tool for capturing interindividual differences in cognitive functioning in epidemiological and aging studies. Dement Geriatr Cognit Disord 7(3):339-345. https://doi.org/10.1159/000479680

Kelter R (2020) Bayesian alternatives to null hypothesis significance testing in biomedical research: a non-technical introduction to Bayesian inference with JASP. BMC Med Res Meth 20(1):142. https://doi.org/10.1186/s12874-020-00980-6

Kliegel M, Martin M, Jäger T (2007) Development and validation of the Cognitive Telephone Screening Instrument (COGTEL) for the assessment of cognitive function across adulthood. J Psychol 141(2):147-170. https://doi.org/10.3200/JRLP.141.2.147-172

Koo TK, Li MY (2016) A guideline of selecting and reporting intraclass correlation coefficients for reliability research. J Chiropract Med 15(2):155-163. https://doi.org/10.1016/j.jcm.2016.02.012

Larner AJ (2021) Cognitive testing in the COVID-19 era: can existing screeners be adapted for telephone use? Neurodegener Dis Manage 11(1):77-82. https://doi.org/10.2217/nmt-2020-0040

Li L, Patel K, Armstrong B, Gallant SN, Wong B (2017) Stereotype threat effects on older adults' memory: a meta-analysis. Innovat Aging 1(supp_1):1064. https://doi.org/10.1093/geroni/igx004. 3894

Liljequist D, Elfving B, Skavberg Roaldsen K (2019) Intraclass correlation: a discussion and demonstration of basic features. PLoS ONE 14(7):e0219854. https://doi.org/10.1371/journal.pone.0219854

Livingston G, Huntley J, Sommerlad A, Ames D, Ballard C, Banerjee S, Brayne C, Burns A, Cohen-Mansfield J, Cooper C, Costafreda SG, Dias A, Fox N, Gitlin LN, Howard R, Kales HC, Kivimäki M, Larson EB, Ogunniyi A, Orgeta V, Ritchie K, Rockwood K, Sampson EL, Samus Q, Schneider LS, Selbæk G, Teri L, Mukadam N (2020) Dementia prevention, intervention, and care: 2020 report of the Lancet Commission. Lancet 396(10248):413-446. https://doi.org/10.1016/S0140-6736(20)30367-6

Lopez MN, Charter RA, Mostafavi B, Nibut LP, Smith WE (2005) Psychometric properties of the folstein Mini-Mental State Examination. Assessment 12(2):137-144. https://doi.org/10.1177/10731 91105275412

Masson MEJ (2011) A tutorial on a practical Bayesian alternative to null-hypothesis significance testing. Behav Res Meth 43(3):679690. https://doi.org/10.3758/s13428-010-0049-5

Mossello E, Boncinelli M (2006) Mini-mental state examination: a 30-year story. Aging Clin Exp Res 18(4):271-273. https://doi. org/10.1007/BF03324660

Myers CE, Radell ML, Shind C, Ebanks-Williams Y, Beck KD, Gilbertson MW (2016) Beyond symptom self-report: use of a computer "avatar" to assess post-traumatic stress disorder (PTSD) symptoms. Stress 19(6):593-598. https://doi.org/10.1080/10253 890.2016 .1232385
Sahakian BJ, Downes JJ, Eagger S, Everden JL, Levy R, Philpot MP, Roberts AC, Robbins TW (1990) Sparing of attentional relative to mnemonic function in a subgroup of patients with dementia of the Alzheimer type. Neuropsychologia 28(11):1197-1213. https:// doi.org/10.1016/0028-3932(90)90055-S

Sahakian BJ, Morris RG, Evenden JL, Heald A, Levy R, Philipot M, Robbins TW (1988) A comparative study of visuospatial memory and learning in Alzheimer-type dementia and parkinson's disease. Brain 111(3):695-718. https://doi.org/10.1093/brain/111.3.695

Sengpiel M, Dittberner D (2008) The computer literacy scale (CLS) for older adults: development and validation. In: Herczeg M, Kindsmüller MC (eds) Mensch and computer 2008. Oldenbourg Wissenschaftsverlag GmbH, München, pp 7-16

Sengpiel M, Jochems N (2015) Validation of the computer literacy scale $(\mathrm{Cls})$. In: Lecture notes in computer science (including subseries lecture notes in artificial intelligence and lecture notes in bioinformatics)

Sindi S, Fiocco AJ, Juster R-P, Pruessner J, Lupien SJ (2013) When we test, do we stress? Impact of the testing environment on cortisol secretion and memory performance in older adults. Psychoneuroendocrinology 38(8):1388-1396. https://doi.org/10.1016/j. psyneuen.2012.12.004

Sprague BN, Freed SA, Phillips CB, Ross LA (2020) A viewpoint on change point modeling for cognitive aging research: moving from description to intervention and practice. Ageing Res Rev 58:101003. https://doi.org/10.1016/j.arr.2019.101003

Stringer G, Couth S, Brown LJE, Montaldi D, Gledson A, Mellor J, Sutcliffe A, Sawyer P, Keane J, Bull C, Zeng X, Rayson P, Leroi I (2018) Can you detect early dementia from an email? A proof of principle study of daily computer use to detect cognitive and functional decline. Int J Geriatr Psychiatr 33(7):867-874. https:// doi.org/10.1002/gps.4863

Vallet F, Mella N, Ihle A, Beaudoin M, Fagot D, Ballhausen N, Baeriswyl M, Schlemmer M, Oris M, Kliegel M, Desrichard O (2020) Motivation as a mediator of the relation between cognitive reserve and cognitive performance. J Gerontol B Psychol Sci Soc Sci 75(6):1199-1205. https://doi.org/10.1093/geronb/gby144

Wechsler D (2008) Wechsler adult intelligence scale-Fourth Edition (WAIS-IV). Pearson, San Antonio

Wilkowska W, Brauner P, Ziefle M (2018) Rethinking technology development for older adults. In: Pak R, Collins McLaughlin A (eds,) Aging, technology and health. Elsevier, pp: 1-30

Publisher's Note Springer Nature remains neutral with regard to jurisdictional claims in published maps and institutional affiliations. 